\title{
ECONOMÍA SOCIAL Y SOLIDARIA EN LA REGIÓN SOACHA - SIBATÉ
}

\author{
Victor Enrique Bonilla Castillo \\ victorbonillacastillo@gmail.com \\ Jaiber Ricardo Díaz Mahecha \\ jdiazmahech@uniminuto.edu.co \\ Corporación Universitaria Minuto de Dios \\ Administración de empresas \\ Colombia
}

Resumen

El trabajo de investigación va enfocado a la pertinencia de la aplicabilidad de la economía solidaria en la región Soacha-Sibaté, se destaca la parte epistemológica con aportes tanto teóricos como empíricos de autores de diferentes nacionalidades y a su vez se evalúan los resultados de las implementaciones del modelo de economía social y solidaria en algunos países, para lograr una economía incluyente, equitativa y participativa.

Se desea dar a conocer los resultados epistémicos y teóricos que por el momento se han encontrado, posteriormente los empíricos, tanto a la población local, posteriormente a la población nacional y aportar al conocimiento regional e internacional sobre una sociedad en la que la prioridad sea entre el sujeto y la armonía con el medio ambiente, generando estrategias con enfoque a lograr la sinergia del ser humano y los recursos naturales, a través de manifestaciones que reconozcas la economía social solidaria como una de las bases del bienestar general de sus habitantes. Claro que también se deben analizar los factores que han hecho que en algunos países la aplicabilidad y los resultados de dicha economía, haya sido muy baja o nula, para evitar los fallos e intentar corregirlos y mejorar las oportunidades obteniendo maximización de sus resultados.

Palabras clave:

Economía solidaria, asociatividad, equidad, política, calidad de vida. 


\section{INTRODUCCIÓN}

Esta investigación se lleva a cabo en dos espacios con los cuales se busca proponer la región Soacha-Sibaté. El primer espacio tiene una superficie de $184.45 \mathrm{~km} 2$, y el segundo $125.60 \mathrm{Km} \mathrm{2.} \mathrm{Así}$ entonces, la región propuesta tiene un espacio de $310 \mathrm{Km} 2$ y una población cercana a 565.393, sin embargo, debido a las circunstancias económicas, demográficas y de migración forzosa, la población en la región ha tenido un crecimiento muy alto.

La economía social y solidaria establece un modelo que busca establecer igualdad de condiciones para todos los miembros de esta región, con el fin de contribuir a la formación y construcción de una sociedad más competitiva, incluyente y equitativa; trabajando en equipo con el fin de mejorar la calidad de vida de todas las personas de la zona. La economía social y solidaria se ha ido construyendo a lo largo del desarrollo y evolución de ciertas sociedades como una alternativa económica, en la cual se establece un modelo que busca establecer igualdad de beneficios y equidad en la distribución de las ganancias de una sociedad, impulsando el desarrollo económico y social, mejorando su nivel y calidad de vida y el entorno en el cual habitan, pues incluye los recursos naturales, los cuales son limitados y en caso de no preservarse, o tomar medidas para su conservación, se vería gravemente afectado el estilo de vida del ser humano, agrega Collin (2008).

Surgen diferentes expectativas de la forma correcta de las manifestaciones económicas en una sociedad basada en una economía social, resultan diversos puntos de partida y algunas aristas que la complementan y la construyen, como el buen vivir que es propuesto por algunas de las culturas originarias, las cuales estaban en una constante búsqueda de una justicia y equidad para todos los miembros de una sociedad, se debe buscar trabajar en equipo de una forma constante con el fin de mejorar la calidad de vida de todas las personas, y lograr un equilibrio entre la producción y el respeto por la naturaleza.

Uno de los principales ejemplos de economía social y solidaria son las cooperativas que surgen de la necesidad de establecer alianzas, uniendo fuerzas, conocimientos, formas de trabajo y producción, con el fin de brindar mejores resultados en la comercialización que requiere una demanda específica, y que una sola persona no los puede suplir, se trabaja en equipo y se busca unificar intereses con el fin de satisfacer más necesidades y lograr mejores objetivos, generando ganancias para todos los miembros de una sociedad y lograr una mejor calidad de vida y un mejor y adecuado desarrollo social(Oviedo \& Silva, 2017).

Así entonces, generar unión de fuerzas y oportunidades con empresas del sector público y privado y que estas no se consideren rivales, se busca generar un trabajo asociado que genere resultados absolutos entre la producción, valor agregado, y generar las mismas posibilidades a todos los grupos de interés, para que los beneficios, resultados y ganancias puedan ser distribuidos de igual forma, en una manera equitativa(Mendoza, 2016).

En cuanto al asociativismo este emerge dentro de una gran variedad de escenarios propuestos por la modernidad como uno de los más antiguos, pues debido a sus prácticas sociales en las que se tienen en cuenta las cuestiones o aspectos generales y específicos, surgió como las primeras prácticas. Se presume que el individuo no siempre se comportó de esta manera, sino que surge como una forma de conseguir poder y realizar un cambio a la civilidad, según Albuquerque, (2003). Así entonces Albuquerque hace énfasis que el proceso en el cual dos o más personas deciden reunirse de forma regular para atender temas o intereses comunes. Se trata de tomar decisiones en las cuales todos estén de acuerdo, aunque haya diferencias, lo ideal es buscar el bien generalizado.

Se destaca la cooperación entre las personas desde sus principales bases como el respeto, reciprocidad, la confianza y no en sentido transaccional o instrumental, en el cual solo se ve al individuo como un objeto que se usa para un fin. De acuerdo al carácter asociativo que tenga lugar entre las personas o grupos, lo importante es dejar en evidencia y aplicar la democracia, en el cual se deja en claro las bases de la acción social de cada grupo, el cual es su razón de ser y de acuerdo a esto, se crea un plan de acción/es, un proyecto que busca como fin, dar solución a las situaciones y superar las dificultades, agrega Albuquerque (2003). 
La constante búsqueda de estrategias para la generación de empleo digno y sustentable, se presenta de manera alterna y capta la atención de los profesionales académicos, despertando su interés para aportar sus conocimientos y así poder establecer un registro, deseando estandarizar sus experiencias y aprendizajes para que se puedan aplicar o enseñar en otros lugares que tengan una situación o problema similar; se analiza y reconoce que esta situación, genera también la importancia de conceptualizar la teoría propuesta como alternativa al capitalismo actual, es de gran importancia la socialización e intercambio entre las diferentes organizaciones, ya que la experiencia adquirida en la práctica puede llevarnos a reforzar nuestras teorías, sustenta Collin (2008).

En la economía solidaria se plantea como uno de los principios la reciprocidad para compartir en lugar de acumular, basándose en la confianza y realizando intercambios. Se devuelve al dinero el fin principal, el cual es ser un medio de intercambio. Los valores que hacen parte de la economía social y solidaria son: Solidaridad, diversidad, equidad, autogestión, ecumenismo (unidad, armonía), comunidad(Huesa, Romero, \& Muñoz, 2016). En la economía social y solidaria, sobresale un concepto, el cual busca fomentar el consumo local de los productos generados en las regiones, dejando de lado lo producido por las grandes industrias nacionales y multinacionales, reduciendo las importaciones, ya que esto ayuda a aumentar el desempleo y obliga a las personas a buscar salarios como medio para comprar productos que anteriormente no eran conocidos o que no necesitaban; por ésta razón la economía solidaria, aunque no es universal o se aplica siempre, existe, para ayudar a la población a mejorar su nivel de vida, agrega entonces Collin (2008).

Coraggio, (2009) explica que las economías son mixtas, puesto que son multiculturales y responden a lógicas distintas, también debido a que de manera simultánea interactúan: el sistema de economía capitalista, pública y popular, a través de sus diferentes asociaciones, por ejemplo: sindicatos, movimientos sociales, ONG, entre otros. Para la economía social solidaria la medida de democratizar la economía es de gran importancia, requiere la inclusión de los grupos de campesinos, artesanos, pescadores, promoviendo una política de autoconsumo y autogestión, lo cual lleva a un modelo auto sostenible que a su vez se puede reproducir, enseñar y afianzar (Benítez, Quintero, Márquez, \& Ayala, 2015; Castro \& García, 2016; Elisondo \& Donolo, 2015; Hernández, 2016; Oviedo \& Silva, 2017).

Se habla sobre una divergencia de pensamientos de unos integrantes y otros, ya que unos pueden ver como prioridad la integración de las personas, aunque no tengan las aptitudes para asumir algunas funciones o tareas, mientras que otros dan más relevancia a la obtención de los resultados, por lo que tienen preferencias por las capacidades y aptitudes o conocimientos de las personas, excluyendo las que no cumplan con el perfil. Es allí cuando se habla de la importancia de construir el conocimiento, donde se capaciten a las personas y puedan aportar en las funciones que se requieren para que el trabajo sea productivo y a su vez se logre mitigar el problema o posibles problemas, de ésta manera, ya no se van a presentar exclusiones, pues las personas estarían en igualdad de condiciones para asumir cualquier función que aporte a la economía social y solidaria, agrega así Coraggio (2009).

La economía solidaria es una alternativa que cuenta con bases que contradicen al sistema económico capitalista. En otras palabras, la economía solidaria es catalogada como una alternativa que combina el que hacer socioeconómico y sociopolítico (Mutuberria, 2008).

La política en sentido positivo según Dussel (2006) es una actividad que organiza y promueve la producción, reproducción y aumento de la vida de miembros. Por lo que aquí se sostiene que esta opción de economía alternativa es disruptiva al sistema capital dominante.

\section{Sobre el territorio: la región}

Para Porto-Goncalves (2008) el territorio ya no solo se visualiza como un componente netamente geográfico, si no es una serie de entramados complejos que involucran a los actores, sujetos, la geografía y su interrelación por sujetos y grupos sociales. Así entonces la región no se 
compone solo de aristas físicas sino también inmateriales, como las manifestaciones laborales, sociales y culturales.

Los sujetos buscan transformar o reconstruir su realidad y sus instituciones respectivas según los propuesto por (Manzanal, 2007). El autor bajo la matriz civilizatoria de la modernidad produce el territorio con una visión que deja a la naturaleza en un plano de instrumento o de recursos al servicio del ser humano. Se puede observar el resultado en la gran crisis ambiental que se viven hoy día.

Los autores principales dentro de la producción social y territorial son tres: el estado, el capital y lo sectores populares, por supuesto cada uno con distintas lógicas. Se menciona que el estado tiene un principio jerárquico territorial de organización, el capital tiene una relación mediada por los flujos de valor, información y producción. Por último, los sectores populares tienen una lógica de búsqueda de condiciones de reproducción de la vida en sociedad, sostiene (Coraggio, 2011).

Se debe retomar la investigación de (Giménez, 1999) donde se hace un análisis de la identidad regional y clasifica los actores en: apáticos y resignados; migrantes potenciales; modernizados; tradicionales y regionalistas. Así mismo es pertinente revisar el proceso de construcción de la subjetividad, ya que es clave para la reconstrucción de la identidad y, por tanto, fundamental para el entendimiento de los conflictos territoriales.

Díaz (2015) indica que las economías solidarias tienden a encontrar en el territorio la proximidad requerida para su desarrollo y la consolidación de mismo frente a las concomías del capital. Actúan bajo el fin de la acumulación ampliada de capital, el estado bajo el funde acumulación de poder político para el ejercicio de gobernabilidad y el sector popular bajo una racionalidad respectiva.

Para poder construir una categoría que ofrezca una relación directa con el territorio, como la región, es preciso visibilizar el hecho de que son distintos colectivos los que tienen el poder económico capitalista y que inclusive entre ellos mismos se encuentran en competencia constante para seguir obteniendo los beneficios económicos del capital, explica Savater (2014).

Así entonces, para el trabajo de esta investigación se propone un territorio como es la región, espacio conformado por dos espacios como lo son Sibaté y Soacha. Territorios que además de tener unas características físicas, tiene unas manifestaciones inmateriales que se representan en la cultura, en la parte social, la parte laboral, entre otras. Dos espacios que, si bien comparten muchas de estas características, de manera individual representan ciertas aristas como en el caso de Soacha que es un territorio conurbado con Bogotá en el cual emerge una población migrante altamente representativa, población laboral migrante tanto nacional como internacional, así como población desplazada debido al conflicto armado en Colombia.

\section{Método}

Se propone llevar a cabo en el proyecto la metodología de Investigación Acción participativa, la cual es una metodología que, aunque reconoce la importancia del aspecto teórico, rescata la importancia de la praxis, de pasar de lo teórico a lo empírico para que esa realidad que viven los actores del escenario económico social de la región aporte a lo teórico. Así entonces, se pretende entrar en un escenario de participación con esa realidad de la población objeto de la investigación, que eventualmente aporta y tiene un significado igual o más relevante que la teoría. No obstante, esta metodología será utilizada cuando se lleve a cabo el trabajo empírico que se hará a través de un trabajo de campo.

Esta metodología es propia de las ciencias sociales, como también para la educación en América Latina, nacida de hecho en Colombia, como es la Investigación Acción Participativa, es propuesta por el colombiano Fals Borda (1987).

Los autores e investigadores que siguen esta metodología han tenido procesos diferentes, sin embargo, vale la pena citar las etapas que Colmenares (2011) identifica y las cuales servirán para la 
realización del proyecto: el diagnóstico, la construcción de planes de acción, la ejecución de dichos planes, y la reflexión permanente de los involucrados en la investigación(Rincón-Báez, Becerra Plaza, Arias-Velandia, \& Durán Becerra, 2018; Luis Martín; Trujillo-Flórez et al., 2019, 2018).

Se define el concepto de ciudad-región, el cual es la interrelación de varias ciudades y su interdependencia para crear un sistema competitivo, para el desarrollo integral tanto de las personas que la integran como del medio ambiente o medio físico que habitan.

Para esta primera parte se establece una estructura de investigación, descriptivo y bibliográfico, en la cual, el primer punto se toma como inicio, la construcción epistemológica y teórica de la economía social y solidaria, para interiorizar todos los términos y contextualizarlos para una mayor comprensión en los conceptos de la aplicación de dicha economía, posterior a ello se realiza la construcción del problema, que ha llevado a que se genere este tipo de diferencias entre un sistema de distribución de riqueza tan desigual e inequitativa en la sociedad en la cual se ve afectada principalmente la sociedad y el medio ambiente, para posteriormente realizar un debate teórico y epistémico, llevando a que las demás personas tomen conciencia de las consecuencias que ha traído la implementación de un sistema que no es justo con las personas de la región y así se pueda implementar a través de un economía solidaria acciones y soluciones a las diferentes situaciones que afectan el nivel de desarrollo de la ciudadanía y su calidad de vida, brindando la oportunidad de desarrollar habilidades que permitan la colaboración y el bienestar común con valores de solidaridad, equidad, reciprocidad, tanto en la parte de conocimiento teórico como en la parte práctica y llegar a dar un enfoque social, en la que el estado tome realmente a la ciudadanía como razón de ser y así mismo como único objetivo, sin dejar de lado los recursos naturales ni anteponiendo intereses personales(Daza-Orozco, 2019; Norman-Acevedo, 2018, 2019).

\section{Resultados}

Coraggio (2009) nos ilustra sobre una investigación en Argentina sobre los procesos de empresas que han sido recuperadas por sus trabajadores, cuando los dueños fundadores de las empresas se declaran en quiebra o la empresa está en proceso de abandono, en cuyo caso es de gran importancia el apoyo no solo de sus empleados, sino de sus familiares y la voluntad para llevar a cabo dicha recuperación; debemos tener presente que los resultados han de ser cambiantes, puesto que también influye la economía en general, las políticas públicas y el sistema de financiamiento. Un ejemplo claro son los emprendimientos asociativos mercantiles, los cuales tienen un estrecho vínculo debido a la zona donde se encuentren o la clasificación del sector económico al que se dedican, utilizando subsidios dispuestos por el estado para fomentar e impulsar el desarrollo y sostenibilidad de los emprendimientos, un punto que demuestra que se deben implementar más políticas de objetivos en el desarrollo de una economía social solidaria, haciéndola democrática y participativa.

Este proyecto de investigación se encuentra en curso, el cual a la fecha está en el alcance de determinar aportes teóricos de la economía social y solidaria y la difusión de los hallazgos, principios y fundamentos de dicha economía, llegando a aumentar los beneficios para las personas implicadas en iniciativas de las mismas características y condiciones, lo cual ha impulsado la implementación de políticas sociales y ambientales que ha mejorado su nivel de vida y a su vez de la región en la cual se aplica.

En los últimos años en diferentes partes del mundo han surgido grupos que consideran que el sistema capitalista ya está desgastado, que no es equitativo y que no ha traído mejoras para la población en su conjunto, solo para unos pocos: los inversores y su capital; es por ello por lo que para las propuestas de una economía nueva se usan términos como: social, solidaria, sustentable, participativa, entre otros términos. Todos apuntan al bien común de la región o zona donde se desea mitigar algún problema puntual, bien sea de salud, educación, vivienda, trabajo, y un nivel de vida digno, Collin (2008), en este caso la región Soacha-Sibaté ubicada en el centro de la cordillera andina de Colombia. 


\section{Discusión y Conclusión}

Se interpretan los resultados de la investigación demostrando la relevancia de la investigación e innovación en el área de conocimiento, el territorio y la distribución territorial va más allá de ser un espacio o una ubicación de referencia únicamente de tipo geográfico, pues lleva inmersa en su esencia características antropológicas, sociales, culturales y se ven permeadas por los aspectos políticos e intereses de las personas, en cuanto a el dominio o poder sobre el mismo y su divergencia entre las personas locales y las personas que pueden influir de manera externa, por ejemplo: a través de acción estatal, Vargas (2012).

Teniendo en cuenta las condiciones, tanto laborales como económicas de Colombia, se puede decir que el modelo que más se ajustaría a las necesidades de ciertas comunidades podría ser la economía solidaria, ya que justamente este modelo es el que más desarrollo ha traído en el país a los grupos con intereses y necesidades socio económicas más vulnerables y de las cuales se tiene información gracias a los diferentes grupos que han adoptado este modelo, según (Santos., 2011), las empresas que más han adoptado este tipo de economía, en Colombia, se han enfocado en procesos de reciclaje y cultivo artesanal, siendo estas personas precisamente las que más dificultades económicas pueden presentar

Desde todas estas bases explicadas a lo largo del texto se pretende establecer la responsabilidad social, la justicia social, dejando de verla como una filantropía. Se busca extender las prácticas de la economía solidaria haciéndola parte de las políticas del estado, establecer cambios en el gobierno que están arraigados en un sistema que ha excluido a sus ciudadanos y que pretende alcanzar la globalización de un método que beneficia a unos pocos; las propuestas de una nueva economía no se van a obtener de un momento a otro, ya que debe tener un tiempo de evolución y mostrar resultados paulatinamente, conviviendo con el sistema actual capitalista; se requiere de la acción, posteriormente la reflexión y el aprendizaje del nuevo sistema con sus propuestas.

Restrepo, Sierra y Bayona (2015) proponen que desde los equipos de semilleros de investigación estudiantes de administración de empresas, aporten realizando estudios de mercadeo para el fortalecimiento del plan de negocios y los procesos de organización, ya que son necesarios para poder implementar, desarrollar y reproducir el sistema de la economía solidaria, logrando un avance social, generando una cultura donde se integren los valores y principios bases para un desarrollo integral de los habitantes a nivel personal, laboral y familiar, generando lazos y relaciones duraderas basadas en el respeto y reciprocidad.

\section{Referencias bibliográficas}

Albuquerque, Paulo (2003). Asociativismo, p.31-38, en La otra economía, editorial Altamira, porto alegre.

Benítez, P., Quintero, M., Márquez, K., \& Ayala, A. O. (2015). Beneficios y garantías de los miembros de la fuerza pública en marcos de justicia transicional en perspectiva comparada: lecciones para el caso colombiano. Panorama, 9(16), 105-116. https://doi.org/10.15765/pnrm.vgi16.630

Bonaventura de Sousa santos (2011). Producir para vivir, los caminos de la producción no capitalista. FCE, México.

Castro, A. M. P., \& García, M. J. M. (2016). CALIDAD Y FORMACIÓN DEL PROFESORADO NOVEL EN LAS UNIVERSIDADES PÚBLICAS GALLEGAS - Quality and training of new teaching staff in Galician public universities. Panorama, 10(19), 8-17. https://doi.org/10.15765/pnrm.v10i19.830

Collin Harguindeguy, Laura (2008). DEl, Departamento Ecuménico de Investigaciones; Alternativas; Consumo; Producción; Ecosol; Economía Social y Solidaria; Sistemas de valores; Economía social; Ética; México

Colmenares, A. (2011). Investigación-acción participativa: una metodología integradora del conocimiento y la acción. Revista Latinoamericana de Educación, Vol. 3, No.1, 102-115.

Coraggio, J. L. (2011). Economía Social y Solidaria. El trabajo antes que el capital. Quito: Ediciones Abya-Yala.

Coraggio, J.L. (2009.) Los caminos de la Economía Social y Solidaria. (Iconos. Revista de Ciencias Sociales. Quito Ecuador.2009). Economista. PhD. (c) Universidad de Pennsylvania.

Cotera, F (2009). Avances y retos de la economía solidaria. Artículo publicado en la Revista América Latina en Movimiento. Recuperado de

DANE (2010). Censo general 2005 Colombia. Bogotá: DANE.

Daza-Orozco, C. E. (2019). Historia de la infancia en el cine colombiano (1st ed.). Retrieved from http://alejandria.poligran.edu.co/handle/10823/1648

Elisondo, R. C., \& Donolo, D. S. (2015). Creatividad y alfabetización informacional. El desafío en cuatro propuestas. Panorama, 8(15), 23-33. https://doi.org/10.15765/pnrm.v8i15.547 
Guerra. P. (2010.) La Economía Solidaria en Latinoamérica. (Pág.67-76) Profesor en la Universidad de la república (Montevideo). Investigador de Economías Solidarias

Guridi, L \& M, C. (2014). La dimensión económica del Desarrollo Humano Local: La economía social y solidaria. San Sebastián, Ed. Hegoa.

Hernández, Y. M. (2016). La Unión Patriótica: memorias para la paz y la democracia. Panorama, 10(18), 27-38. https://doi.org/10.15765/pnrm.v10i18.822

Huesa, A. A. R., Romero, R. F., \& Muñoz, D. P. G. (2016). La formación en ciudadanía en escenarios de educación inicial: una experiencia con madres comunitarias. Panorama, 10(18), 102-119. https://doi.org/10.15765/pnrm.v10i18.826

Mendoza, J. A. G. (2016). Empresa privada: principal socio en el posconflicto y la construcción de la paz. Panorama, 10(18), 8492. https://doi.org/10.15765/pnrm.v10i18.823

Norman-Acevedo, E. (2018). Rompiendo Barreras, 10 Años de la Educación Virtual en el Politécnico Grancolombiano. (primera; Eduardo Norman-Acevedo, ed.). Retrieved from http://alejandria.poligran.edu.co/handle/10823/1146

Norman-Acevedo, E. (2019). Consumer cultural studies. Bogotá: Institución Universitaria Politécnico Grancolombiano.

Oviedo, L. B., \& Silva, M. C. (2017). LA INVESTIGACIÓN ACCIÓN Y EL APRENDIZAJE POR PROYECTOS EN EL MARCO DEL MODELO PEDAGÓGICO ENSEÑANZA PARA LA COMPRENSIÓN. EXPERIENCIA DEL COLEGIO VISIÓN MUNDIAL EN COMUNIDADES VULNERABLES DE MONTERÍA-Action research and Project Based Learning in ... Panorama, 11(21), 38-51. https://doi.org/10.15765/pnrm.v11i21.1053

Porto-Goncalves (2009). De Saberes y de Territorios: diversidad y emancipación a partir de la experiencia latinoamericana. Polis, Revista de la Universidad Bolivariana, Volumen $8, \mathrm{~N}^{\circ} 22$, Venezuela.

Restrepo, Blanca; Sierra, Jose; Bayona, María, (2015). Caracterización socioeconómica de soacha de la población vulnerable de altos de cazucá del municipio de soacha (cundinamarca) y la economía solidaria., Colombia, 2015, pp. 69-79, Revista santo tomás ediciones USTA universidad santo tomás

Rincón-Báez, W. U., Becerra Plaza, G. E., Arias-Velandia, N., \& Durán Becerra, E. (2018). Inteligencia de Datos en la formación en Administración y Negocios en Colombia 2018 (Vol. 1; Institución Universitaria Politécnico Grancolombiano, ed.). https://doi.org/10.1017/CBO9781107415324.004

Rodríguez, C. (2004). En busca de alternativas económicas en tiempos de globalización: el caso de las cooperativas de recicladores de basura en Colombia, en Boaventura de Sousa Santos y Mauricio García Villegas (eds.) Emancipación social y violencia en Colombia. Bogotá: Norma.

Rofman, A. (2010). La economía solidaria y los desafíos actuales. Revista de ciencias sociales, No.18, 159-175.

Trujillo-Flórez, Luis Martín; Bernal Yermanos, M. Á., Escobar Castro, J. A., González Triana, M. C., Gutiérrez Carvajal, J. P., Gutiérrez Vanegas, J. C., ... Torres López, A. L. (2019). Experiencias de innovación educativa - Tomo 3 (1st ed.; Luis Martín Trujillo-Flórez, ed.). Bogotá: Politécnico Grancolombiano.

Trujillo-Flórez, Luis Martín; Martínez Contreras, R. M., Espitia López, H., Rojas Paredes, J. C., Vargas Leguizamón, Y. R., \& Castro Cabal, G. A. (2018). Experiencias de innovación educativa (1st ed.; L. M. Flórez-Trujillo, ed.). Bogotá: Politécnico Grancolombiano.

Vargas Ulate, Gilbert (2012). ESPACIO Y TERRITORIO EN EL ANÁLISIS GEOGRÁFICO. Reflexiones, vol. 91, núm. 1, pp. $313-326$ Universidad de Costa Rica San José, Costa Rica.

www.alainet.org/es/active/38210. 